\title{
Oxidative stress markers may not be early markers of doxorubicin-induced cardiotoxicity in rabbits
}

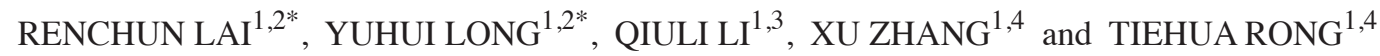 \\ ${ }^{1}$ State Key Laboratory of Oncology in South China, Guangzhou; Departments of ${ }^{1}$ Anesthesiology, \\ ${ }^{2}$ Head and Neck Oncology, and ${ }^{3}$ Thoracic Surgery, Sun Yat-Sen University Cancer Center, Guangzhou, P.R. China
}

Received April 18,2011; Accepted June 6, 2011

DOI: $10.3892 /$ etm.2011.306

\begin{abstract}
This study was performed to determine whether oxidative stress markers may be early markers of doxorubicininduced cardiotoxicity. Forty-four male rabbits were randomly divided into four doxorubicin groups and one control group ( 8 rabbits). The control group received saline, and rabbits in the doxorubicin groups received $2 \mathrm{mg} / \mathrm{kg}$ doxorubicin weekly for 1 (group 1, 8 rabbits), 2 (group 2, 8 rabbits), 4 (group 3, 9 rabbits) or 8 (group 4, 11 rabbits) weeks. Echocardiography was performed to measure left ventricular ejection fraction, fractional shortening and the E/A ratio. Cardiotoxicity scores were determined by light microscopy using Billingham's method and by electron microscopy. Serum glutathione peroxidase (GPx) and superoxide dismutase (SOD) concentrations were quantified by a rabbit-specific enzyme-linked immunosorbent assay (ELISA). The Billingham cardiomyopathy scores for the rabbits in groups 3 or 4 were significantly higher $(\mathrm{p}<0.05)$ compared to the scores for the control group or groups 1 and 2. Myocardial injury was demonstrable by electron microscopy in groups 2,3 and $4(\mathrm{p}<0.05)$. Serum GPx concentrations decreased only in group 4 compared to the control group $(\mathrm{p}<0.05)$. No changes were measured in serum SOD concentration. The results indicate that oxidative stress markers may not be early markers of doxorubicininduced cardiotoxicity.
\end{abstract}

\section{Introduction}

Anthracycline antibiotics, such as doxorubicin, are potent antitumor agents used in a wide spectrum of malignancies $(1,2)$. However, the use of anthracyclines is limited by the risk of developing life-threatening congestive heart failure $(3,4)$. The early detection of anthracycline-induced cardiotoxicity is crucial for the prevention of heart failure.

Correspondence to: Dr Tiehua Rong, State Key Laboratory of Oncology in South China, Guangzhou, P.R. China

E-mail: lairch@sysucc.org.cn

${ }^{*}$ Contributed equally

Key words: oxidative stress markers, doxorubicin, cardiotoxicity
Although myocardial biopsy is the 'gold standard' for evaluating anthracycline-induced cardiotoxicity, the invasiveness of this procedure excludes it from routine clinical use (5). Recently, many studies concerning early biochemical markers for detecting doxorubicin-induced cardiotoxicity have been performed $(6,7)$.

The major hypothesis regarding the pathophysiology of anthracycline-induced cardiotoxicity is that cardiac damage is caused by oxidative stress through the generation of reactive oxygen species (ROS) $(8,9)$. The reduction of several NADPH-dependent microsomal enzymes by doxorubicin results in ROS generation. Mitochondria are a primary target of doxorubicin-induced cardiotoxicity mediated by the induction of ROS. This increase in ROS leads to several damaging events in the mitochondrion that drastically diminish mitochondrial function. The enzymatic antioxidant system consists of superoxide dismutase (SOD) and glutathione peroxidase (GPx), which inactivate ROS. These enzymes indirectly reflect ROS activity. Few studies have been carried out on oxidative stress markers for the early detection of doxorubicin-induced cardiotoxicity $(10,11)$. Thus, the aim of the present study was to evaluate whether oxidative stress markers may be early markers of doxorubicin-induced cardiotoxicity.

\section{Materials and methods}

Model of doxorubicin-induced cardiomyopathy. Forty-four male rabbits used in this study were maintained in an airconditioned room, fed with commercial standard chow and allowed free access to tap water ad libitum in the Laboratory Animal Center of Sun Yat-Sen University. All experimental procedures were approved by the Center for Drug Evaluation and Research Institutional Animal Care and Use Committee and were in compliance with the Guidelines for the Use and Care of Laboratory Animals. Rabbits were randomly divided into one control group (8 rabbits) and four doxorubicin groups. The rabbits in the control group received weekly intravenous injections of saline comparable to the volume of doxorubicin received by rabbits in the doxorubicin groups. Rabbits in the doxorubicin groups received intravenous injections of $2 \mathrm{mg} /$ kg doxorubicin (Shenzhen Main Luck Pharmaceuticals, Inc., Shenzhen, China) via an ear marginal vein at weekly intervals for 1 (group 1, 8 rabbits), 2 (group 2, 8 rabbits), 4 (group 3, 9 rabbits) or 8 (group 4, 11 rabbits) weeks. Animals were 


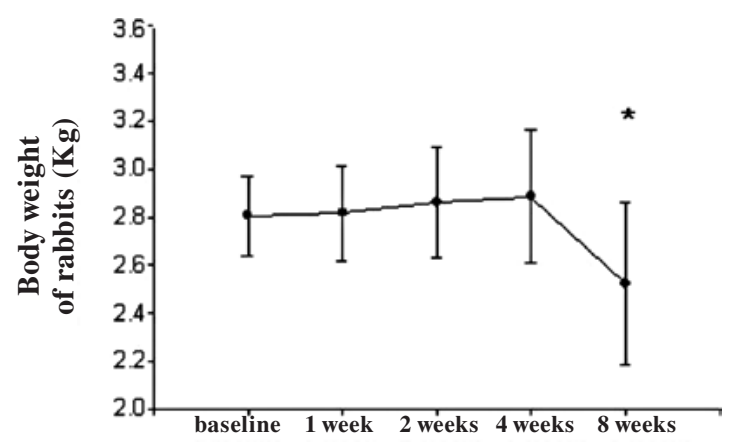

Figure 1. Changes in rabbit mean weight during doxorubicin-induced cardiotoxicity. Data are presented as the means $\pm \mathrm{SD}$. ${ }^{*} \mathrm{p}<0.05$ vs. the control group.

observed daily and weighed weekly throughout the duration of the study.

Pathological evaluation of the heart. Two weeks after the designated number of doses had been given, the rabbits were euthanized with $3 \%$ pentobarbital sodium administered into a marginal ear vein. As soon as ethically possible, the chest cavity was opened and the left ventricle harvested and processed as described below, according to a previously reported protocol (12). Animals that died spontaneously during the study were not included in the data analysis.

One portion of the harvested left ventricle was fixed in phosphate-buffered $10 \%$ formalin, embedded in paraffin and sectioned at a thickness of $5 \mu \mathrm{m}$. Sections were stained with H\&E. The frequency and severity of doxorubicin-induced myocardial lesions were assessed semi-quantitatively by light microscopy.

Cardiac samples from 4 rabbits in each group were processed for electron microscopy. In these rabbits, a portion of the left ventricle was rapidly (within $1 \mathrm{~min}$ ) cut into $1-\mathrm{mm}^{3}$ pieces, fixed with $2.5 \%$ glutaraldehyde and stored at $4^{\circ} \mathrm{C}$. These portions were embedded in glycol methacrylate resin, sectioned at a thickness of $1 \mu \mathrm{m}$ and stained with alkaline toluidine blue for electron microscope analysis. Changes were graded based on the number of myocytes showing myofibrillar loss and cytoplasmic vacuolization [score of 0-3 according to Billingham (13)] in the toluidine blue-stained sections.
Serum GPx and SOD concentrations. To monitor serum GPX and SOD concentrations, blood samples were collected before dosing and 2 weeks after the administration of 1,2,4 or 8 weekly doses of doxorubicin. Blood samples were collected from the marginal artery of either ear. Blood samples were centrifuged at $2,000 \mathrm{rpm}$ for $10 \mathrm{~min}$ at $4^{\circ} \mathrm{C}$, and the sera was frozen at $-80^{\circ} \mathrm{C}$ until assayed. The blood samples from the rabbits that died were excluded from the analysis. Serum GPX and SOD concentrations were assayed using a rabbit NT-proBNP specific enzyme-linked immunosorbent (ELISA) assay.

Statistical analysis. Data were reported as the means \pm standard deviation (SD). One-way analysis of variance (ANOVA) was used to determine significant differences in the rabbits' weight. The Mann-Whitney test for non-parametric data was used to determine significant differences in cardiomyopathy scores. The Tukey-Kramer multiple comparisons test was used to assess significant differences in GPx or SOD concentrations among the groups. A p-value $<0.05$ was considered statistically significant.

\section{Results}

General toxicity. Four doxorubicin-treated rabbits died during the 9-week experimental period (1 after a cumulative dose of $8 \mathrm{mg} / \mathrm{kg}$, and 3 after a cumulative dose of $16 \mathrm{mg} / \mathrm{kg}$ ). No arterial blood samples for GPx and SOD analysis were available from these animals, and they were excluded from the study. Thus, a total of 8 rabbits were analyzed in group 3, and 8 rabbits in group 4. Compared to the control group, the rabbits in group 4 experienced a loss in body weight ( $\mathrm{p}<0.05$, Fig. 1).

Myocardial pathology. Doxorubicin caused myocardial damage that was visible by light microscopy as cytoplasmic vacuolization and loss of myofibrils at all dose levels. Both changes were frequently noted in the same cell, and the frequency of affected cells increased as lesions became more severe. Data on the incidence and severity of these lesions at the various cumulative doses of doxorubicin are summarized in Table I. Electron microscopy revealed three types of myocardial damage: sarcoplasmic vacuolization, mitochondrial swelling and disruption, and myofibrillar lysis. Mitochondrial

Table I. Cardiomyopathy scores.

\begin{tabular}{lcccccc}
\hline & No. of animals & \multicolumn{4}{c}{ Cardiomyopathy score } \\
\cline { 3 - 6 } & & 0 & 1 & 1.5 & 2 & 2.5 \\
\hline Control group & 8 & 8 & 0 & 0 & 0 & 0 \\
Group 1 & 8 & 7 & 1 & 0 & 0 & 0 \\
Group 2 & 8 & 4 & 3 & 1 & 0 & 0 \\
Group 3 & 8 & 0 & 1 & 4 & 3 & 0 \\
Group 4, & 8 & 0 & 0 & 0 & 1 & 0 \\
\hline
\end{tabular}

Cardiomyopathy scores were based on the percentage of myocytes showing cytoplasmic vacuolization and/or myofibrillar loss, and graded from 0 to 3 as follows: 0 , no damaged cells; $1,<5 \% ; 1.5,5-15 \% ; 2.0,16-25 \% ; 2.5,26-35 \%$; and 3, $>35 \%$ damaged cells. ${ }^{a}$ Cardiomyopathy scores significantly higher $(\mathrm{p}<0.05)$ than those in the control group and groups 1 and 2 (Mann-Whitney test). ${ }^{\mathrm{b}}$ Cardiotoxicity scores significantly higher $(\mathrm{p}<0.05)$ than those in the control group and groups 1,2 and 3 (Mann-Whitney test). 


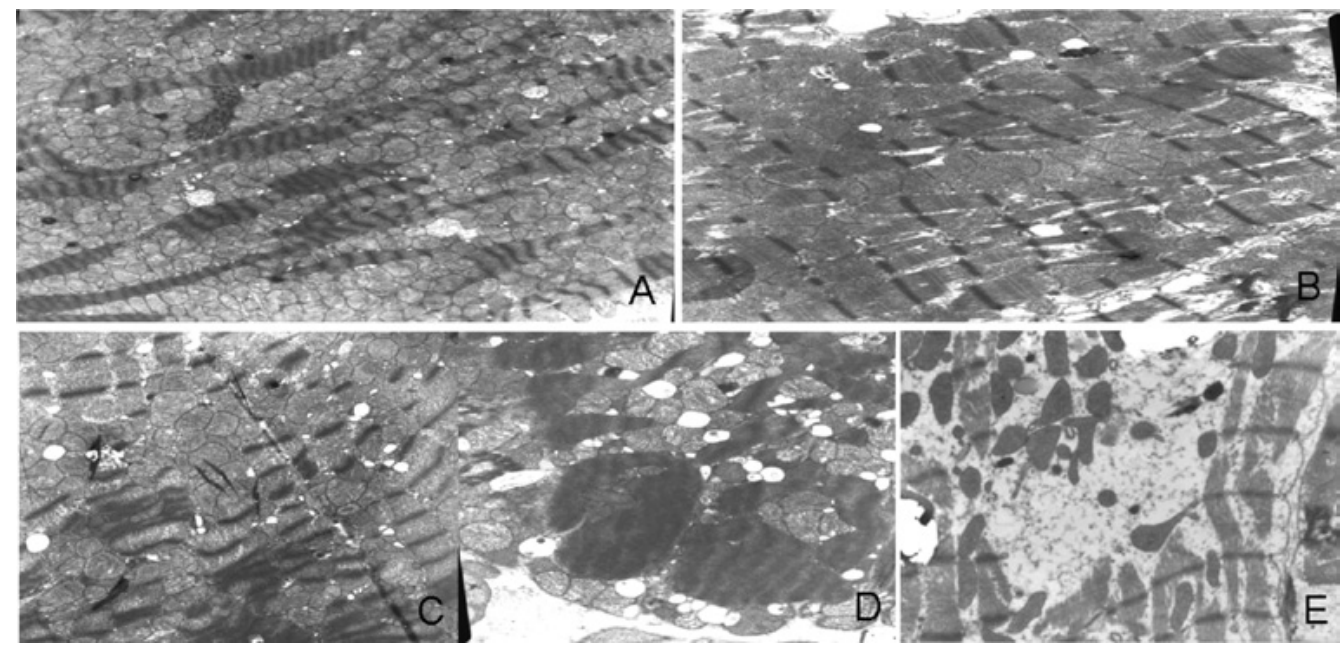

Figure 2. Changes in the heart as assessed with electron microscopy. (A) Normal mitochondria and myofibrils in the control group; $x 4,500$. (B) Mitochondria and myofibrils in rabbits receiving a cumulative doxorubicin dosage of $2 \mathrm{mg} / \mathrm{kg} ; \mathrm{x} 4,500$. (C) Early stage degeneration in cardiac muscle cells in rabbits receiving a cumulative doxorubicin dosage of $4 \mathrm{mg} / \mathrm{kg} ; \mathrm{x} 4,500$. The sample shows partial myofibril lysis with hydropic distension of the sarcoplasmic reticulum and T-tubules, as well as swollen and disrupted mitochondria. (D) Extensive myofibril lysis and disrupted mitochondria in rabbits receiving a cumulative doxorubicin dosage of $8 \mathrm{mg} / \mathrm{kg} ; \mathrm{x} 4,500$. (E) Myofibril lysis and disrupted mitochondria in rabbits receiving a cumulative doxorubicin dosage of $16 \mathrm{mg} / \mathrm{kg} ; \mathrm{x} 9,600$.

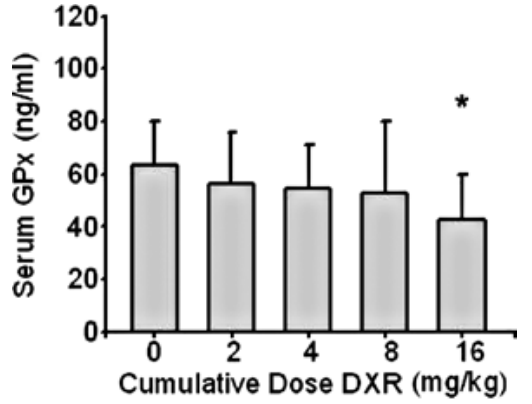

Figure 3. Changes in the serum GPx concentration. Data are presented as the means \pm SD. ${ }^{*}$ p $<0.05$ vs. the control group.

swelling and myofibrillar lysis occurred in groups 2, 3 and 4. As the cumulative dose of doxorubicin increased, the mitochondrial disruption and lysis of myofibrils also became more serious (Fig. 2).

Serum GPx concentration. The mean serum GPx concentration in the control group was $63 \pm 17 \mathrm{ng} / \mathrm{ml}$. Compared to the control group, serum GPx concentrations decreased in group 4 $(\mathrm{p}<0.05)$ (Fig. 3).

Serum SOD concentration. The mean serum SOD concentration in the control group was $2.4 \pm 1.7 \mathrm{ng} / \mathrm{ml}$. No significant differences were found between the control group and groups 1 , $2,3$ or 4 ( $p>0.05)$ (Fig. 4).

\section{Discussion}

Anthracyclines, particularly doxorubicin and epirubicin, are among the most commonly used cytotoxic drugs because of their potent anticancer activity. However, the use of anthracyclines is limited due to the risk of irreversible congestive heart failure. Due to poor prognosis, the early detection of doxorubicin-induced cardiotoxicity is very important. Pre-clinical anthracycline cardiomyopathy remains disputed. Many

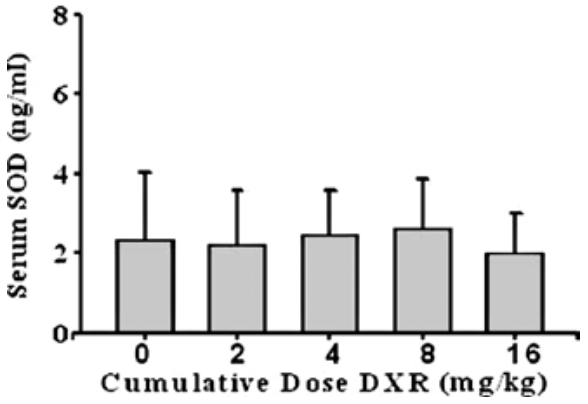

Figure 4. Changes in serum SOD concentrations. Data are presented as the means $\pm \mathrm{SD}$

studies have shown that mitochondrial swelling or disruption and myofilament lysis is the result of anthracycline-induced cardiotoxicity $(14,15)$, but no definitive electron microscope grading scheme has been developed. In this study, we found that rabbits receiving cumulative doxorubicin dosages of $4 \mathrm{mg}$ / $\mathrm{kg}$, despite a normal appearance under the light microscope, had mitochondrial swelling or disruption and myofibril lysis visible with electron microscopy.

Several studies support the notion of mitochondria being the main source of doxorubicin-induced ROS. Doxorubicininduced free radicals would lead to lipid peroxidation and membrane damage (16). Changes in membrane permeability and subsequent cardiomyocyte destruction are the final steps of cell damage. The cardiotoxic effect of doxorubicin-induced cardiotoxicity has been attributed to irreversible damage of mitochondria in heart cells, which express a unique enzyme on the inner membrane that is able to reduce anthracyclines to their semiquinone derivatives. Increased oxidative stress and an antioxidant deficit have been suggested to play a major role in doxorubicin-induced cardiomyopathy and congestive heart failure (17). ROS, mainly superoxide anions, hydrogen peroxide and hydroxyl radical, are too unstable to measure in the serum. The enzymatic antioxidant system consists of SOD and GPx, which indirectly reflect ROS activity. In this study, 
cardiac injury was demonstrable by electron microscopy at the cumulative dose of $4 \mathrm{mg} / \mathrm{kg}$ doxorubicin. However, no changes in serum GPx or SOD concentration were found at the cumulative dose of $4 \mathrm{mg} / \mathrm{kg}$ doxorubicin. Mercuro et al (10) found that oxidative stress parameters may play a role as useful markers of early cardiotoxicity, but they did not obtain a myocardial biopsy. This finding may explain the different results to some extent.

Classical long-term models have been applied by several researchers (18). The rabbit model is considered the basis for comparing new anthracyclines and new cardioprotective compounds, generally over periods of 10-18 weeks, with cumulative doses of $20-30 \mathrm{mg} / \mathrm{kg}$. Thus, the classical longterm rabbit model was used for early and rapid evaluation of anthracycline cardiotoxicity. In this study, the rabbits became weak or died after 8 weeks of $2 \mathrm{mg} / \mathrm{kg}$ doxorubicin weekly. Thus, the duration was limited to only 8 weeks.

This study had several limitations. First, the mean follow-up period of the different groups was very short, so the possibility that a longer-term study may find greater damage in the higher dose groups and result in higher GPx or SOD concentrations cannot be excluded. Second, the sample size was small. A larger study with a longer follow-up period after high cumulative doses of anthracycline is required to address these issues.

In conclusion, increased cumulative anthracycline doses are not associated with an early and significant increase in oxidative stress. The predictive value of serum GPx and SOD concentrations for the development of cardiomyopathy is not yet known and should be evaluated in a prospective follow-up study.

\section{References}

1. Torrisi R, Cardillo A, Cancello G, et al: Phase II trial of combination of pegylated liposomal doxorubicin, cisplatin, and infusional 5-fluorouracil (CCF) plus trastuzumab as preoperative treatment for locally advanced and inflammatory breast cancer. Clin Breast Cancer 10: 483-488, 2010.

2. Krischer JP, Epstein S, Cuthbertson DD, Goorin AM, Epstein ML and Lipshultz SE: Clinical cardiotoxicity following anthracycline treatment for childhood cancer: the Pediatric Oncology Group experience. J Clin Oncol 15: 1544-1552, 1997.

3. Alvarez JA, Scully RE, Miller TL, Armstrong FD, Constine LS, Friedman DL and Lipshultz SE: Long-term effects of treatments for childhood cancers. Curr Opin Pediatr 19: 23-31, 2007.
4. Li K, Sung RYZ, Huang WZ, et al: Thrombopoietin protects against in vitro and in vivo cardiotoxicity induced by doxorubicin. Circulation 113: 2211-2220, 2006.

5. Jemal A, Siegel R, Ward E, Murray T, Xu J, Smigal C and Thun MJ: Cancer statistics, 2006. CA Cancer J Clin 5: 106-130, 2006.

6. Horacek JM, Vasatova M, Ticky M, Pudil R, Jebavy L and Maly J: The use of cardiac biomarkers in detection of cardiotoxicity associated with conventional and high-dose chemotherapy for acute leukemia. Exp Oncol 32: 97-99, 2010.

7. Horacek JM, Ticky M, Jebavy L, Pudil R, Ulrychova M and Maly J: Use of multiple biomarkers for evaluation of anthracycline-induced cardiotoxicity in patients with acute myeloid leukemia. Exp Oncol 30: 157-159, 2008.

8. Faber M, Coudray C, Hida H, Mousseau M and Favier A: Lipid peroxidation products, and vitamin and trace element status in patients with cancer before and after chemotherapy, including adriamycin. A preliminary study. Biol Trace Elem Res 47: 117-123, 1995.

9. Kaya E, Keskin L, Aydogdu I, Kuku I, Bayraktar N and Erkut MA: Oxidant/antioxidant parameters and their relationship with chemotherapy in Hodgkin's lymphoma. J Int Med Res 33: 687-692, 2005.

10. Mercuro G, Cadeddu C, Piras A, et al: Early epirubicin-induced myocardial dysfunction revealed by serial tissue Doppler echocardiography: correlation with inflammatory and oxidative stress markers. Oncologist 12: 1124-1133, 2007.

11. Horino N, Kobayashi Y and Usui T: Elevation of lipid peroxide in children treated with a combination of chemotherapeutic agents including doxorubicin. Acta Paediatr Scand 72: 549-551, 1983.

12. Lai RC, Wang XD, Zhang X, Lin WQ and Rong TH: Heart fatty acid-binding protein may not be an early biomarker for anthracycline-induced cardiotoxicity in rabbits. Med Oncol: Feb. 10, 2011 (E-pub ahead of print).

13. Billingham ME, Mason JW, Bristow MR and Daniels JR: Anthracycline cardiomyopathy monitored by morphologic changes. Cancer Treat Rep 62: 865-872, 1978.

14. Wallace KB: Doxorubicin-induced cardiac mitochondrionopathy. Pharmacol Toxicol 93: 105-115, 2003.

15. Berthiaume JM and Wallace KB: Adriamycin-induced oxidative mitochondrial cardiotoxicity. Cell Biol Toxicol 23: 15-25, 2007.

16. Milei J, Boveris A, Llesuy S, Molina HA, Storino R, Ortega D and Milei SE: Amelioration of adriamycin-induced cardiotoxicity in rabbits by prenylamine and vitamins $\mathrm{A}$ and $\mathrm{E}$. Am Heart J 111: 95-102, 1986

17. Sun $X, Z$ hou $Z$ and Kang YJ: Attenuation of doxorubicin chronic toxicity in metallothionein-overexpressing transgenic mouse heart. Cancer Res 61: 3382-3387, 2001.

18. Van Vleet JF and Ferrans VJ: Clinical and pathologic features of chronic adriamycin toxicosis in rabbits. Am J Vet Res 41: 1462-1469, 1980. 